\title{
Treatment of Avascular Necrosis of the Proximal Pole of the Scaphoid by Arthroscopic Resection and Prosthetic Semireplacement Arthroplasty Using the Pyrocarbon Adaptive Proximal Scaphoid Implant (APSI): Long-Term Functional Outcomes
}

\author{
Mathilde Gras, MD ${ }^{1}$ Abhijeet L. Wahegaonkar, MBBS, DOrtho, DNB (Ortho), MS, MCh (Ortho) $)^{2,3}$
} Christophe Mathoulin, MD ${ }^{4}$

1 Institut de l'Appareil Locomoteur Nollet, Paris, France

2 Upper Extremity, Hand and Microvascular Reconstructive Surgeon,

Hand Surgery Associates, Pune, Maharashtra, India

3 Sancheti Institute for Orthopedics and Rehabilitation, Pune,

Maharashtra, India

${ }^{4}$ Institut de la Main, Clinique Jouvenet, Paris, France

J Wrist Surg 2012;1:159-164

Address for correspondence and reprint requests Prof. Christophe Mathoulin, MD, Institut de la Main, Clinique Jouvenet, 6 square Jouvenet, 75016 Paris, France (e-mail: cmathoulin@orange.fr).

\begin{abstract}
Keywords

- avascular necrosis

- non-union

- proximal pole scaphoid

- pyrocarbon implant

- wrist arthroscopy

Fractures of the proximal pole of the scaphoid with associated avascular necrosis and nonunion are well known to be problematic. Many techniques for fixation and reconstruction of the proximal pole of the scaphoid have been reported, often with poor results. One of the newer modalities of treatment for these difficult cases is excision of the proximal pole and replacement with a pyrocarbon implant. The ovoid shape of the implant acts as a spacer and repositions itself throughout the range of motion of the wrist. The procedure can be performed arthroscopically, thus reducing the morbidity and allowing the procedure to be a day case. Satisfactory results have been reported in elderly patients, but there is a paucity of literature regarding the outcomes in younger patients. This multicenter retrospective study evaluates the clinical, radiologic, and functional outcomes in patients under the age of 65 years with a minimum follow up of 5 years. There were 14 patients with scaphoid nonunion advanced collapse (SNAC) grade I, II, or III wrists, with a mean age of 53 years and mean follow up of 8.7 years. There were improvements in all of the patient related variables including VAS pain scores $(7.5$ to 0.7$)$, extension $\left(45^{\circ}\right.$ to $\left.60^{\circ}\right)$, flexion $\left(32^{\circ}\right.$ to $53^{\circ}$ ), and grip strength ( 15.8 to $34.6 \mathrm{~kg}$ ). Complications included volar subluxation of the implant, which was acutely surgically corrected (1), volar subluxation of the implant, with persistent pain that required a four-corner fusion (2), and secondary radial styloidectomy (3). This technique is an attractive, minimally invasive alternative for nonunion and avascular necrosis resulting from fractures of the proximal pole of the scaphoid. The authors provide details of the technique, including technical suggestions for performing the procedure.
\end{abstract}


The treatment of nonunion of proximal pole of the scaphoid, with avascular necrosis, is still challenging. The geometry of the scaphoid, the direction and type of fracture, and the vascular pattern to the scaphoid all influence the decision making and optimum treatment of these fractures. Fractures proximal to the nutrient vessels entering on the dorso-radial surface can result in ischemia of the proximal pole. These fractures are often diagnosed late, frequently resulting in nonunion. The nonunion can occur with or without osteonecrosis. The natural history is poor, with progression to radioscaphoid arthritis and carpal collapse, called scaphoid nonunion advanced collapse (SNAC), also known as the SNAC wrist.

The most commonly advocated procedure for the treatment of the SNAC wrist in the early stages is a vascularized pedicled bone graft. ${ }^{1-3}$ When it is no longer possible to reconstruct the scaphoid, a salvage procedure is indicated. Based on the grade of arthritis, different procedures, ranging from a proximal row carpectomy to partial wrist arthrodesis, have been described in literature. ${ }^{4-9}$ When the necrotic proximal pole cannot be reconstructed, however, and if the cartilage of the scaphoid fossa of the radius is still intact, replacing the proximal pole with an implant may be an alternative. Many such implants/prostheses have been reported, but more recently a pyrolytic carbon (pyrocarbon) implant, which replicates and adapts to the carpal kinematics very closely, has demonstrated good clinical outcomes by limiting the progression of carpal collapse. ${ }^{10}$ The recent advances in wrist arthroscopy have made it possible to insert this implant with a minimally invasive technique, thus reducing the morbidity of the procedure. ${ }^{11}$ Excellent results have been reported in elderly patients using this technique. ${ }^{12}$ This study was designed to analyze the clinical, radiological, and functional outcomes in functionally demanding patients less than 65 years of age and with a minimum follow-up of 5 years.

\section{Nonsurgical Treatment of Scaphoid Nonunion}

The diagnosis of a scaphoid fracture is based on clinical suspicion, a supporting history, physical examination, and imaging studies. In the acute injury the patient typically presents with low-grade wrist pain after a fall onto an extended wrist. A history of high energy trauma fall should raise the suspicion of a concomitant carpal injury. In an established nonunion with arthritic changes, the patient presents with decreased wrist motion, weakness, inability to perform pushups, and radial-sided wrist pain.

Nonoperative management options include the use of a wrist splint, physiotherapy and avoidance of aggravating factors. Adams et $\mathrm{al}^{13}{ }^{13}$ however, reported that nonoperative treatment is not as effective as surgical intervention and typically is not recommended for managing scaphoid nonunion, even though there are reports indicating that some nonunions remain stable or nondisplaced and free of arthritis even after 10 years. ${ }^{14,15}$ The natural history of scaphoid nonunion has been defined, and there is a correlation between nonunion and osteoarthritis. As a consequence, even in patients who are relatively asymptomatic, surgery is recommended. ${ }^{16-19}$

\section{Surgical Treatment Options for Scaphoid Nonunion}

Ideally the preferred option would be to fix the proximal pole of the scaphoid. However, if this is not possible, then a reconstructive procedure is required. A simple resection of the proximal pole of the scaphoid could be performed, but there is a concern regarding the stability of the carpus. ${ }^{20}$ Many different surgical reconstructive options for SNAC wrist have been reported. Several papers have reported using a synthetic replacement such as silicone, Vitallium, ${ }^{21}$ acrylic $^{22,23}$ and titanium, ${ }^{24}$ glutaraldehyde and bovine fibrocartilage, ${ }^{25}$ and collagen and bone morphogenetic protein (BMP). ${ }^{26}$ The popular salvage procedures include a fourcorner fusion and a proximal row carpectomy. The technique that the authors prefer, however, is a minimally invasive option, which can be performed as a day case, and does not preclude proceeding with other secondary surgical options.

\section{Materials and Methods}

\section{The Adaptive Proximal Scaphoid Implant}

The Adaptive Proximal Scaphoid Implant (APSI) (BioprofileTornier) is composed of pyrolytic carbon (pyrocarbon), which is inert and biocompatible and has been used in heart valves for decades. ${ }^{12}$ It has a low coefficient of friction, and its modulus of elasticity is similar to that of bone. It allows smooth motion and loading with minimal secondary degeneration. The implant is an ovoid shape that allows adaptive mobility during motion. In the frontal plane the smaller radius of curvature corresponds to the scaphoid fossa, and in the transverse plane the larger radius of curvature is directed anteroposteriorly and the smaller radius of curvature lies in the frontal plane. These two axes of the implant confer on it adaptability to the kinematics of the wrist (-Fig. 1). Because of this three-dimentional reorientation

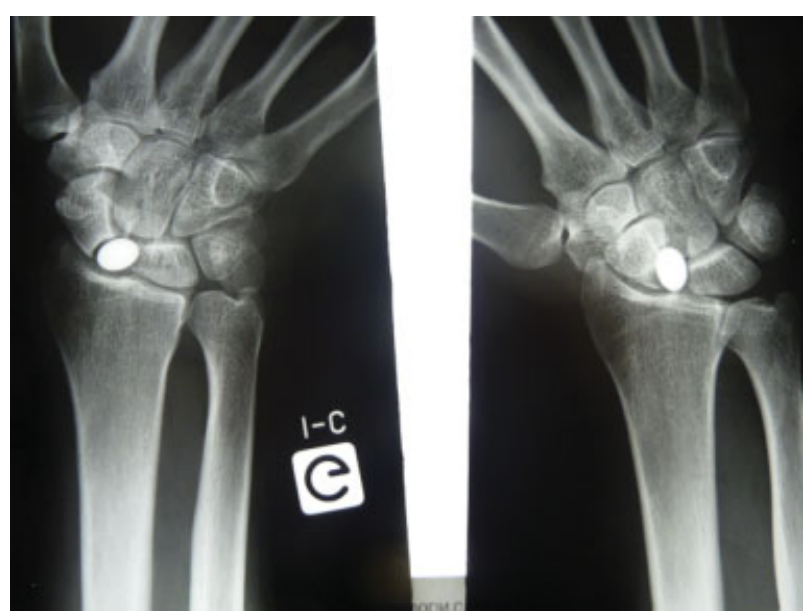

Fig. 1 Position of the APSI during radial and ulnar deviation of the wrist. 


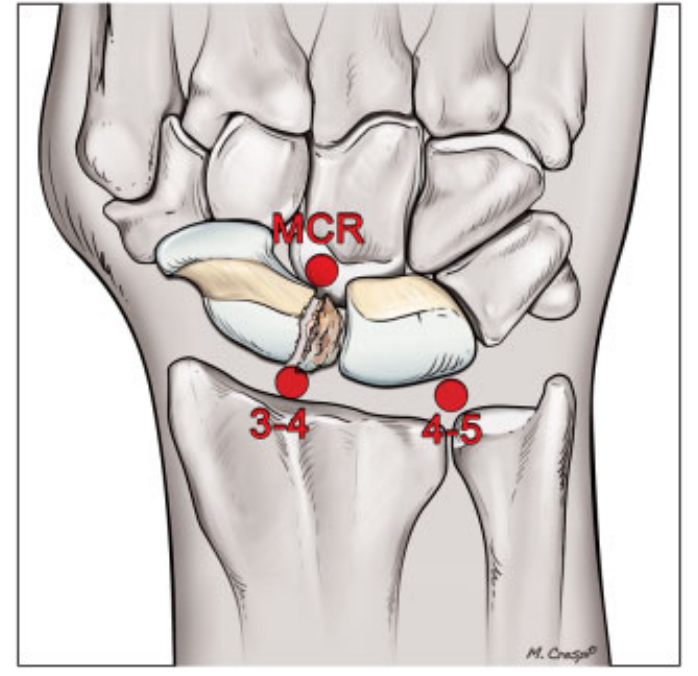

Fig. 2 The three usual portals for arthroscopic proximal pole replacement.

during the movements of the wrist, the implant remains stable in the physiological range and does not require any form of fixation or soft tissue reinforcement. There are three commercially available implant sizes: small, medium, and large.

\section{Surgical Technique (Video 1)}

The procedure is performed as a day surgery under regional anesthesia and tourniquet control. The arm was placed on a table with a countersupport. Longitudinal traction is applied on the fingers using Chinese finger traps, usually with 4 to $6 \mathrm{~kg}$. The arthroscope is usually placed in the 4-5 portal, but the $6 \mathrm{R}$ portal is also used at times, allowing optimal visualization to locate the proximal pole and any associated injuries. The, the 3-4 portal is established under vision, which allows for direct access to the nonunion (-Fig. 2). Thereafter, a radial mid-carpal portal is made to assess the status of the cartilage and for proper placement of the implant. The scapholunate ligament, which is still attached to the proximal pole, is now divided with a number 15 scalpel through the 3-4 portal. Once divided, the proximal pole of the scaphoid is removed with a grasping forceps or a fine hemostat through the 3-4 portal ( - Fig. 3a, b). A full-radius small-diameter shaver may also be used to facilitate resection of the proximal pole. The size of the implant is determined by comparing the size of resected proximal pole to trial implants (-Fig. 4). A trial implant is then inserted. If it is not well seated, a larger implant is inserted. It is subsequently removed and replaced with the definitive prosthesis under arthroscopic control. If necessary, in SNAC I and II, a styloidectomy should be performed. A final arthroscopic assessment should be performed of the radiocarpal and midcarpal joints to check the correct position of implant ( $\mathbf{- F i g . 5}$ ). The arthroscope is then removed, and the stability of the implant is assessed under fluoroscopy in all positions of the wrist. The authors leave the small portals open, except the 3-4 portal, which is extended to insert the implant and is closed with a single suture. A semicompressive bulky dressing is applied for a few days.

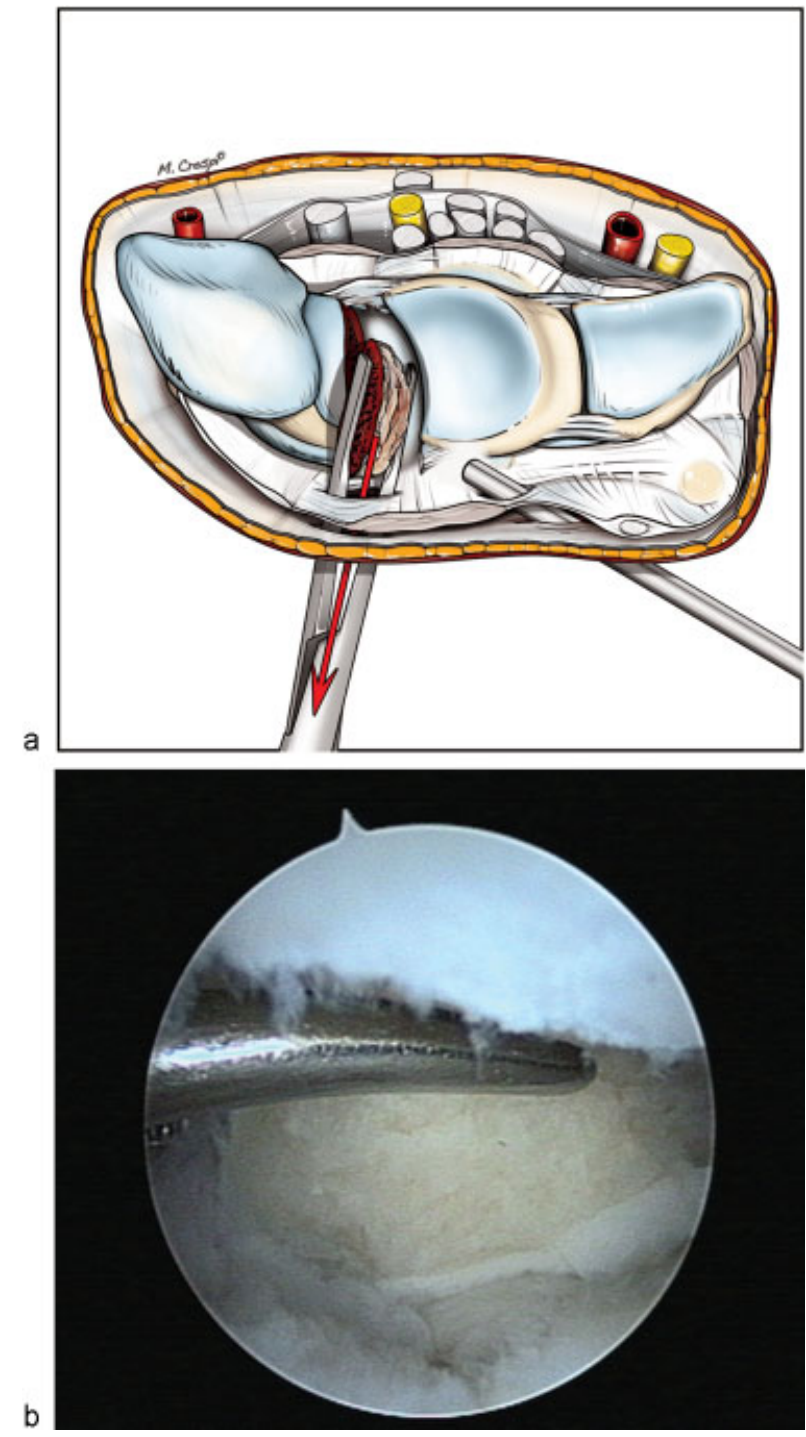

Fig. 3a, b (a) Diagram and (b) arthroscopic radiocarpal view showing the resection of the proximal pole of the scaphoid.

\section{Postoperative Protocol and Rehabilitation}

Mobilization is allowed immediately. The patient performs range of motion (ROM) exercises at home as instructed by the physical therapist. The dressing is changed in the office at 5-7 days after surgery and is usually discarded at this time and the sutures are removed. Progressively increasing ROM exercises are encouraged for 4-6 weeks postoperatively, followed by strengthening exercises.

\section{Pearls}

- Judiciously use the 4-5 and 6R portals for assessment and debridement of the wrist.

- Use a number 15 scalpel blade or arthroscopic scissors to cut the scapholunate ligament. Ensure that the ligament is completely released to facilitate the excision of the proximal pole fragment.

- Use a fine hemostat to grasp the fragment prior to removal. The remaining distal scaphoid needs to be fashioned in such a way to help accommodate the implant 


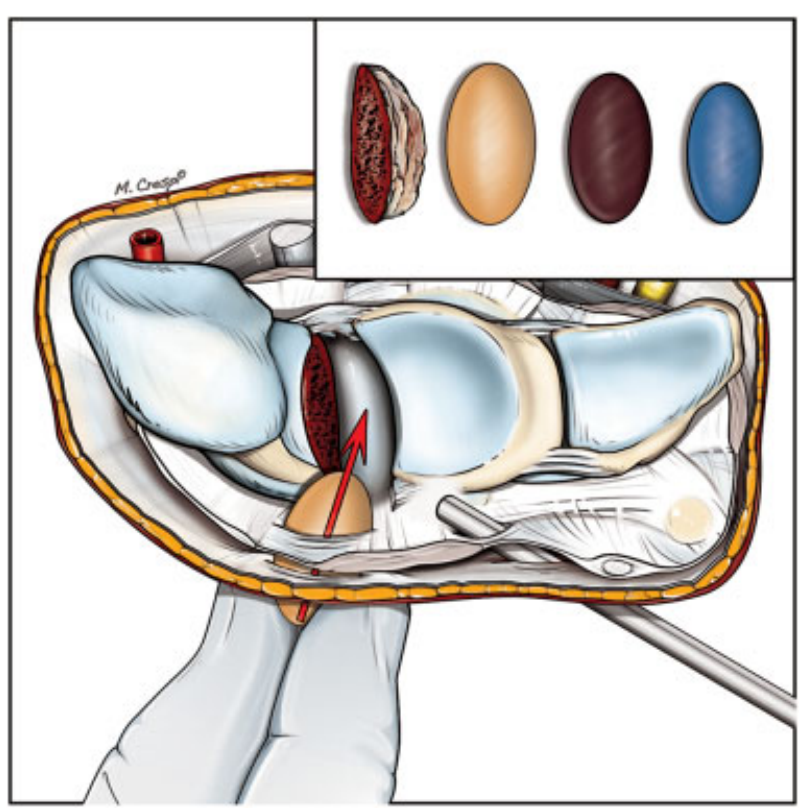

Fig. 4 Determination of correct implant size with the three different implant sizes and the resected proximal scaphoid.

- Use a trial implant to assess the stability in all directions of motion.

- Styloidectomy should be performed in each case with SNAC I, to avoid secondary procedures.

\section{Pitfalls}

- Avoid injury to cutaneous nerves.

- Avoid injury to the extensor tendons when releasing the scapholunate ligament with the scalpel.

- Avoid injury to the volar capsule.

\section{Personal Series}

A retrospective study was performed on all patients under 65 years of age who underwent an arthroscopic APSI procedure, which included 15 patients, all operated on by the

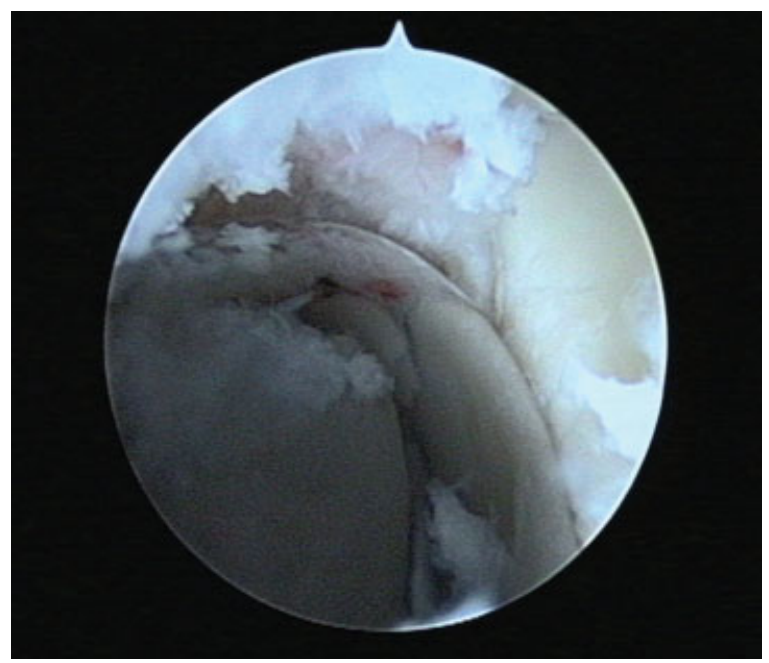

Fig. 5 Midcarpal arthroscopic view showing the correct position of the implant

\section{Video 1}

This video shows all stages of the implementation of the APSI implant to replace the necrotic proximal pole of scaphoid. The views are of the radiocarpal joint, with the scope in the 4-5 portal and the instruments in the 3-4 portal. In the midcarpal joint the scope is in the radial midcarpal portal. With the first arthroscopic pass the radiocarpal joint is assessed and debrided, and the scapholunate interosseous ligament is incised with a scalpel. Following removal of the proximal scaphoid, different implant trials are inserted. In this video, the small blue trial prosthesis is inserted, then the large yellow trial prosthesis, and finally the medium brown trial prosthesis. The definitive prosthesis is inserted and confirmed to be correctly positioned from both the radiocarpal and midcarpal joints.

Online content including video sequences viewable at: www.thieme-connect.com/ejournals/html/10.1055/ s-0032-1329591

senior author (CM) between February 1999 and January 2006. One patient was lost to follow-up, leaving a total of 14 patients. All patients in this study were men who presented with avascular necrosis of the scaphoid following nonunion of the proximal pole. The average age was $52.7 \pm 9.9$ years (range 40-65 years) with an average follow-up of $8.7 \pm$ 1.8 years (range $6-11$ years). Of these patients, $43 \%$ were manual workers. Of the nonunions, $71 \%$ involved the dominant wrist.

A review of the preoperative histories revealed that 7 of the 14 patients were unaware of any injury to the wrist. The other seven had a mean delay from injury to surgery of 21.1 years (range 3-40 years). Two cases (14.3\%) were initially treated nonoperatively. Four patients (28.6\%) had a failed Matti-Russe procedure. One of these four patients had a silicone prosthesis replacement of the proximal pole of the scaphoid.

The preoperative radiological assessment revealed that all patients had a nonunion of the proximal pole with avascular necrosis. Two patients (14\%) had a fracture of the body of the scaphoid, and 12 patients ( $86 \%$ ) had a fracture of the proximal pole. Arthritic changes corresponded to Grade I SNAC wrist in four cases (29\%); Grade II SNAC wrist in seven cases (50\%), and Grade III SNAC wrist in three cases (21\%). Among the patients with SNAC II and III, eight cases (57\%) had a dorsal intercalated segment instability (DISI) deformity.

The postoperative immobilization for pain control averaged $25.4 \pm 10.4$ days (range 10 to 45 days). All patients had the option of removing their splint to use their hand and wrist, if they wished. The mean follow-up was $8.7 \pm 1.7$ years (range, 6 to 11 years). Two patients who initially presented with a fracture of the body of the scaphoid returned later with a subluxation of the implant. Both underwent a four-corner fusion at 6 and 24 months, respectively, for persistent pain.

Of the 12 other patients who retained their implants, there was one patient with an early volar subluxation of the implant identified on the eighth postoperative day, which 
was managed with a radial styloidectomy and repositioning of the implant. Three other patients required a radial styloidectomy at 2-3 years after the initial procedure. Therefore, $36 \%$ of patients required a radial styloidectomy.

The Visual Analog Scale (VAS) pain scores (0-10) improved from 7.5 preoperatively to 0.7 postoperatively. The average objective measures improved from preoperative to postoperative, with contralateral in brackets: extension $45^{\circ}$ to $60^{\circ}$ $\left(72^{\circ}\right)$; flexion, $32^{\circ}$ to $53^{\circ}\left(64^{\circ}\right)$; radial deviation, $7^{\circ}$ to $18^{\circ}$ $\left(24^{\circ}\right)$; ulnar deviation, $20^{\circ}$ to $30^{\circ}\left(37^{\circ}\right)$; grip strength, 15.8 to $34.6(44.1) \mathrm{kg}$.

On long term radiological follow-up, all patients had a stable implant. Notching of the capitate was evident on plain radiographs in six patients. The DISI was corrected in five cases (42\%) ( - Fig. 6a-d); but it persisted in one patient ( $8 \%$ ).

The functional results of patients were evaluated using the Modified Mayo Wrist Score (MMWS), which averaged $79.6 \pm 4$ (range 75-85). The results were excellent to good in $66.7 \%$ of the cases and average in $33.3 \%$ of the cases. The average Disabilities of the Arm, Shoulder, and Hand (DASH) score was $7.6 \pm 2.3$ (range 2.3 to 15.9 ).

In the two patients who initially presented with a fracture of the body of the scaphoid associated with a Grade III SNAC wrist and a DISI deformity, the pain persisted and we found a subluxation of the implant in one of the patients. A salvage procedure of four-corner arthrodesis was performed at 6 months in one patient and at 2 years in another. In these patients, the MMWS scores were 25 and 55 respectively, and the DASH scores were 50 and 43.2 respectively, accounting for our poor results and failures.

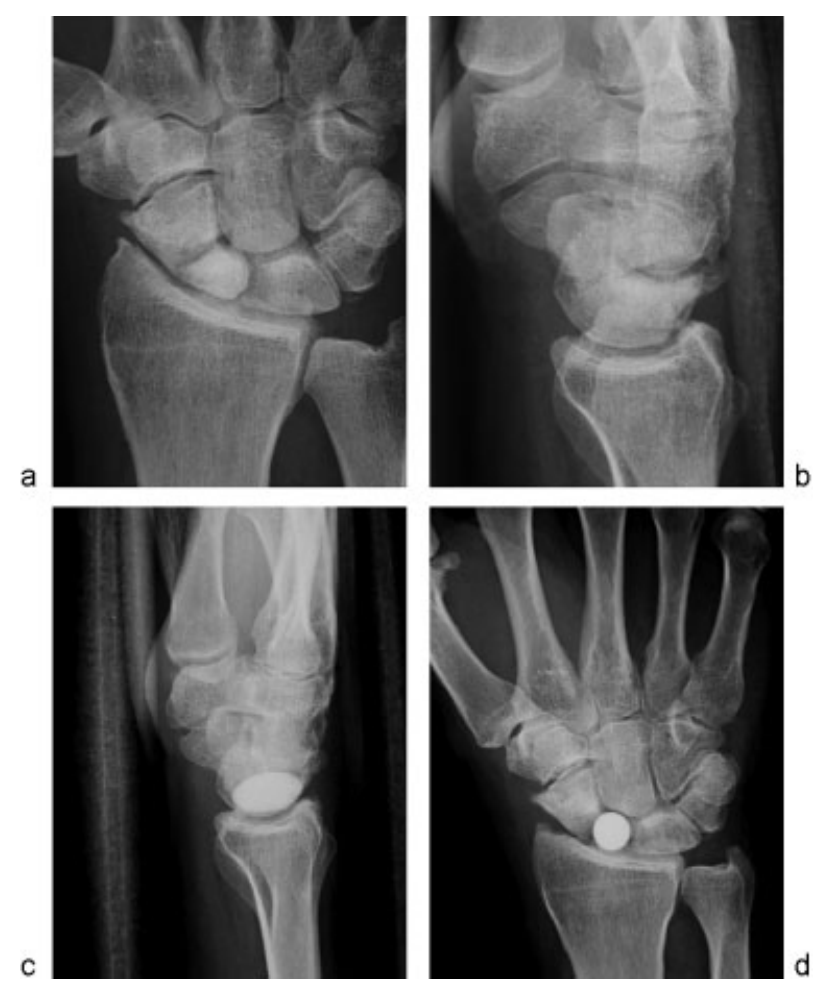

Fig. 6a-d Clinical case: (a, b) Necrosis of proximal pole with adaptative DISI. (c, d) Radiographs after 10 years showing the correct position of implant, the efficient styloidectomy, and the corrected DISI.

\section{Discussion}

Satisfactory results in the long term with respect to carpal stability, reliability and reproducibility of functional results, and overcoming pain can be achieved by arthroscopy-assisted pyrocarbon implant arthroplasty in the treatment of avascular necrosis of the proximal pole of the scaphoid. The clinical and radiological results are satisfactory, and a radial styloidectomy appears advisable. However, the use of the implant for a nonunion of the body of the scaphoid gave poor results, necessitating a palliative surgery for two such cases in our series.

When union of the scaphoid is no longer possible, some authors propose a simple resection of the proximal pole of the scaphoid. ${ }^{27}$ Several implants have also been proposed. Silicone prosthesis for the proximal pole was proposed by Swanson. The long-term results ${ }^{20,28}$ are satisfactory in terms of pain control and function; however, the rate of revision surgery for secondary dislocation of the implant is relatively large, in addition to a high rate of cystic bone changes secondary to a synovitis caused by the silicone implant. ${ }^{29}$ Furthermore, this implant does not prevent the evolution of osteoarthritis. Silicone replacement, therefore, has been gradually abandoned. Other implants have also been suggested, and they include Vitallium, ${ }^{21}$ acrylic, ${ }^{22,23}$ and titanium, ${ }^{24}$ which too has fallen out of favor. Some authors have also proposed a composite implant made of glutaraldehyde and bovine fibrocartilage ${ }^{25}$ or of collagen and bone morphogenetic protein (BMP), ${ }^{26}$; with unsatisfactory results.

In 2000, Pequignot et $\mathrm{al}^{10}$ reported the APSI with satisfactory functional results at an average follow-up of 6 years. No dislocation or instability of the implant was noted, and in all cases, the carpal height was maintained. This technique appears to be promising in the prevention of carpal collapse in scaphoid nonunions. In 2007, Grandis and Berzero ${ }^{30}$ confirmed these results in a retrospective study of 98 patients aged 22 to 65, who had an implant replacement using the APSI for the proximal pole of the scaphoid, with satisfactory outcomes, with respect to pain relief, return to work, and improved range of movement and strength, with 79 patients being satisfied with the procedure.

\section{References}

1 Kakar S, Bishop AT, Shin AY. Role of vascularized bone grafts in the treatment of scaphoid nonunions associated with proximal pole avascular necrosis and carpal collapse. J Hand Surg Am 2011; 36(4):722-725, quiz 725

2 Merrell GA, Wolfe SW, Slade JF III. Treatment of scaphoid nonunions: quantitative meta-analysis of the literature. J Hand Surg Am 2002;27(4):685-691

3 Mathoulin C, Gras M, Roukos S. Vascularized bone grafting from the volar distal radius for carpal bones reconstruction [in French]. Chir Main 2010;29(Suppl 1):S65-S76

4 Tünnerhoff HG, Haussmann P. Partial midcarpal arthrodesis with excision of the scaphoid for the treatment of advanced carpal collapse [in German]. Oper Orthop Traumatol 2005;17(3):233-248

5 Merrell GA, McDermott EM, Weiss AP. Four-corner arthrodesis using a circular plate and distal radius bone grafting: a consecutive case series. J Hand Surg Am 2008;33(5):635-642 
6 Ho PC. Arthroscopic partial wrist fusion. Tech Hand Up Extrem Surg 2008;12(4):242-265

7 Buote NJ, McDonald D, Radasch R. Pancarpal and partial carpal arthrodesis. Compend Contin Educ Vet 2009;31(4):180-192

8 Zeplin PH, Kuhfuss I. Midcarpal arthrodesis with cortical bolting chip for treatment of grade II/III scaphoid non-union and scapholunate advanced collapse [in German]. Handchir Mikrochir Plast Chir 2009;41(3):183-185

9 Dimitrios G, Athanasios K, Ageliki K, Spiridon S. Capitolunate arthrodesis maintaining carpal height for the treatment of SNAC wrist. J Hand Surg Eur Vol 2010;35(3):198-201

10 Pequignot JP, Lussiez B, Allieu Y. An adaptive proximal scaphoid implant [in French]. Chir Main 2000;19(5):276-285

11 Mathoulin C. Arthroscopic arthroplasty for proximal pole scaphoid nonunion. In: Geissler WB, ed. Atlas of the Hand Clinics. Philadelphia, PA: WB Saunders; 2001:341-358

12 Tian CL, Hetherington VJ, Reed S. A review of pyrolytic carbon: application in bone and joint surgery. J Foot Ankle Surg 1993; 32(5):490-498

13 Adams BD, Frykman GK, Taleisnik J. Treatment of scaphoid nonunion with casting and pulsed electromagnetic fields: a study continuation. J Hand Surg Am 1992;17(5):910-914

14 Ruby LK, Stinson J, Belsky MR. The natural history of scaphoid nonunion. A review of fifty-five cases. J Bone Joint Surg Am 1985; 67(3):428-432

15 Mack GR, Bosse MJ, Gelberman RH, Yu E. The natural history of scaphoid non-union. J Bone Joint Surg Am 1984;66(4):504-509

16 Cooney WP, Linscheid RL, Dobyns JH, Wood MB. Scaphoid nonunion: role of anterior interpositional bone grafts. J Hand Surg Am 1988;13(5):635-650

17 Fernandez DL. Anterior bone grafting and conventional lag screw fixation to treat scaphoid nonunions. J Hand Surg Am 1990; 15(1):140-147

18 Nakamura R, Imaeda T, Tsuge S, Watanabe K. Scaphoid non-union with D.I.S.I. deformity. A survey of clinical cases with special reference to ligamentous injury. J Hand Surg [Br] 1991;16(2):156-161
19 Stark A, Broström LA, Svartengren G. Surgical treatment of scaphoid nonunion. Review of the literature and recommendations for treatment. Arch Orthop Trauma Surg 1989;108(4): 203-209

20 Vinnars B, Adamsson L, af Ekenstam F, Wadin K, Gerdin B. Patientrating of long term results of silicone implant arthroplasty of the scaphoid. Scand J Plast Reconstr Surg Hand Surg 2002;36(1): 39-45

21 Leslie BM, O'Malley M, Thibodeau AA. A forty-three-year followup of a Vitallium scaphoid arthroplasty. J Hand Surg Am 1991; 16(3):465-468

22 Orsi R, De Tullio V, Brunelli F. Results 37 years after insertion of an acrylic implant for scaphoid pseudoarthrosis [in French]. Ann Chir Main Memb Super 1995;14(4-5):214-217

23 Barber HM. Acrylic scaphoid prostheses: a long-term follow up. Proc R Soc Med 1974;67(10):1075-1078

24 Swanson AB, de Groot Swanson G, DeHeer DH, et al. Carpal bone titanium implant arthroplasty. 10 years' experience. Clin Orthop Relat Res 1997;(342):46-58

25 Heatley FW, Allen PR. Glutaraldehyde treated bovine fibrocartilage arthroplasty for the scaphoid: a case history and discussion. Biomaterials 1986;7(4):305-307

26 Kujala S, Raatikainen T, Ryhänen J, Kaarela O, Jalovaara P. Composite implant of native bovine bone morphogenetic protein (BMP) and biocoral in the treatment of scaphoid nonunions-a preliminary study. Scand J Surg 2002;91(2):186-190

27 Garcia-Elias M, Lluch A. Partial excision of scaphoid: is it ever indicated? Hand Clin 2001;17(4):687-695, x

28 Haussman P. Long-term results after silicone prosthesis replacement of the proximal pole of the scaphoid bone in advanced scaphoid nonunion. J Hand Surg [Br] 2002;27(5):417-423

29 Peimer CA, Medige J, Eckert BS, Wright JR, Howard CS. Reactive synovitis after silicone arthroplasty. J Hand Surg Am 1986; 11(5):624-638

30 Grandis C, Berzero GF. Partial scaphoid pyrocarbon implant: personal series review. J Hand Surg [E] 2007;32(supplement 1):95 